\title{
Towards an Intelligent Approach for the Restitution of Physical Soil Parameters
}

\author{
Ibtissem HOSNI ${ }^{1}$, Lilia BENNACEUR FARAH ${ }^{2}$ \\ Laboratory of Remote Sensing and Information Systems \\ with Spatial Reference, ENIT, Tunis El Manar University \\ Tunis, Tunisia
}

\author{
Imed Riadh FARAH ${ }^{3}$ \\ Laboratory of Software Engineering Research \\ Distributed Applications, Decisional Systems and Intelligent \\ Imaging ENSI, La Manouba University, Tunis, Tunisia
}

\author{
Raouf BENNACEUR ${ }^{4}$ \\ Laboratory of Physics of Condensed Matter \\ Tunis, Tunisia
}

Mohamed Saber NACEUR 5

Laboratory of Remote Sensing and Information Systems with Spatial Reference, ENIT, Tunis El Manar University

Tunis, Tunisia

\begin{abstract}
The analysis of the radar response on natural surfaces has been subject of intense research during the last decades in the field of remote sensing. Unless the availability of accurate values of surface roughness parameter, the restitution of soil moisture from radar backscattering signal can constantly provide inaccurate estimates. Characterization of soil roughness is not fully understood, so a wide range of roughness values can be obtained for the same studied surface when using different measurement methodologies. Various studies have shown a weak agreement between experimental measurements of soil physical parameters and theoretical values under natural conditions. Due to this nonlinearity and its ill-posedness, the inversion of backscattering radar signal on soils for restitution of physical soil parameters is particularly complex. The aim of the present work is the restitution of soil physical parameters from backscattered radar signal using an adapted backscattering model to the soil proposed description. As our study focuses on little rough soils, we have adopted in this work a multi-layered modified multiscale bi-dimensional Small Perturbation Model (2D MLS SPM). Subsequently, we propose a new way of describing the dielectric constant, with the aim of including air fractions in the multiscale multilayer description of the soil. Calculating the dielectric constant is based on the consideration of a soil comprising two phases, a fraction of soil, and an air fraction. For the inversion method, a methodology of coupling between neural networks (NN) and genetic algorithms (GA) was carried on in order to restitute the physical properties of the soil. Samples were generated by the original MLS 2D SPM followed by a neural network to obtain the statistic soil moisture and MLS roughness parameters algorithm. Thereafter, these restored values were modelled by the genetic algorithms to resolve, in part or in whole, the disagreement between the retrieval and original values.
\end{abstract}

Keywords-Inversion; air fractions; multi-layered; multiscale; SPM; genetic algorithms

\section{INTRODUCTION AND BACKGROUND}

Although soil moisture shows only a small proportion of the amount of water on Earth [12], it plays an extremely important role in different environmental sciences. This parameter, closely related to the soil dielectric constant, is strongly involved in the regulation of evapotranspiration phenomenon which directly affects atmospheric dynamics [10]. Modelling of this phenomenon is mainly related to a better understanding spatial organization structure of humidity.

Soil roughness plays an important role in the capture of water [13] [14] [15], promoting its infiltration and reducing its downward flow [1]. Therefore, the measurement of soil roughness should be taken into account to study and model the processes of runoff and erosion of agricultural land. It would also be very useful for better understanding the hydrodynamics and soils drainage.

Being two extremely dynamic variables under natural conditions, the possibility of achieving their estimations by means of remote sensing observations is very interesting for many applications [16].

Many studies have focused on the interest of radar remote sensing to characterize the soil and its parameters [11] [17] [18].

In this context, previous works [15], have characterized natural surfaces as stationary random processes where the distribution of heights defining the roughness of the soil is considered to be the superposition of a finite number of fractal one-dimensional Gaussian processes, each with a different spatial scale [8].

In the literature, a fractal surface model, where multiscale roughness is represented by two new parameters $v$ and $\gamma 0$ related respectively to the fractal dimension $D$ and to the standard deviation of heights $s$ [15], has been synthesized using the wavelet transform for one-dimensional surfaces.

However, to describe soil surfaces, one-dimensional profiles are insufficient because the roughness varies in all directions. Thus, in the context of this work, we have extended this multiscale description to bi-dimensional multilayered surfaces using the adapted bi-dimensional wavelet transform and the Mallat multi-resolution algorithm [2] [4], with the particularity of the presence of air pockets in the soil volume structure and its impact on the backscattering radar signal. 
This paper is organized into two parts.

First part will carry the research methodology and it's organized into four sections.

The first section describes the bi-dimensional multiscale description of natural rough surfaces.

Section 2 shows the radar remote sensing opportunities for proposed natural soil characterization.

The third section deals with the direct problem, that is to study the SPM model adapted to the proposed description of the natural soil and calculate the received radar signal for a given atmosphere conditions and with a given surface geometry.

Section 4 discusses the opposite problem, which consists in estimating the geophysical soil parameters from remote sensing data obtained from the direct model.

The second part describes the results of the selected inversion algorithm in the restitution of physical soil parameters, namely the multiscale roughness and moisture, from the radar signal.

\section{RESEARCH METHODOLOGY}

\section{A. Multiscale Description of a Natural Soil}

A natural soil is generally described by its dielectric properties as well as its roughness which is directly related to the geometry of the surface. Conventionally, natural surfaces are described by two statistical parameters calculated from micro-topography profiles: a vertical component representing the elevation of the surface characteristics above and below its mean level, and a horizontal component explaining the lateral spacing between these characteristics. Generally, the first component corresponds to the amplitude and is expressed as a mean square deviation of heights (RMS for Root Mean Square), the second component represents horizontal variability and is associated with a correlation length (denoted $L_{C}$ ). The characterization of a surface only by one or the other parameter results in an incomplete description of the roughness. The larger RMS is, the greater vertical variations of the surface. The computation of $L_{C}$ supposes to determine the autocorrelation function noted ACF, which expresses the correlation between pairs of measurement points separated by a distance $\Delta$. It varies between -1 and 1 and, by definition, reaches its maximum for a distance $\Delta=0$. It tends to decrease when $\Delta$ increases. The correlation length is then given by the value of $\Delta$ for which $\mathrm{ACF}$ fall to $e^{-1}$ (about 37\%) times its maximum value. The larger $L_{C}$ is, the less the surface is showing horizontal variations.

Further characterization of roughness requires the consideration of its multiscale nature. Many studies [9] have shown that most natural surfaces can be described by a selfaffine (or fractal) statistic according to fractal Brownian motion theory over a wide range of scales ranging from micrometer to kilometer [5]. In the case of a self-affine surface, an increase of the scale by an $f$ factor in the horizontal direction results in a scale change $f^{H}$ in the vertical direction $(H$ is called the Hurst coefficient and quantifies the roughness change rate with the scale), and this to maintain the statistic characterization of the surface.

This assumption involves a single spatial scale for the surface characterization and its statistical properties by computing its standard deviation of heights and correlation length.

In this work, we consider the distribution of heights describing the soil roughness as the superposition of monodimensional Gaussian fractal processes each having a different spatial scale [6]. To describe this multiscale roughness in the case of bi-dimensional surfaces, we use the parameters $v$ and $\gamma 0$ respectively related to the fractal dimension and the standard deviation of heights using the bi-dimensional wavelet transform and the Mallat multi-resolution algorithm [2] [4], to better describe natural surfaces.

\section{B. Radar Backscattering Modeling by a Rough Surface}

1) Proposed description of the studied soil: It's about describing the characteristics of a natural soil through its roughness and its dielectric permittivity. The rough surface, being considered stationary and ergodic in mean and variance, its roughness is entirely defined by the distribution and autocorrelation function of its heights. The second characteristic parameter is its water content. This is usually determined using the dielectric constant of the medium, a function of the soil moisture content, its composition, its temperature and the frequency of observation of the medium.

Conceptually, a multilayered approach was selected for the soil description and its reflection, since there are not really any physical layers but rather a continuous dielectric variability. A simplified technique was chosen for the volume. We proposed the use of an SPM surface diffusion model while considering the surface permittivity. We used for the upper layer a 2D multiscale description of soil roughness using the wavelet transform and the Mallat algorithm [2] [4]. The lower subsurface layer is divided into three fictitious layers separated by an assumed plane interface. We then calculated an effective resulting permittivity which includes the different dielectric permittivities of the three layers.

2) Introduction of air pockets in the soil volume structure: Because of the porosity characteristic of the soil, air can circulate through it, it's the aeration of the soil. More pores are numerous, well organized in network (good distribution between macro-pores and micro-pores, strong connections between the pores), more the air circulate easily towards the deep layers. A well oxygenated soil favors the mineralization of the organic matter in assimilable elements by the plant, and allows the breathing of the living organisms.

Interior works [7] modified the expression of the dielectric constant to take in consideration the presence of air pockets in the soil volume structure. The presence of air fractions is an influencing factor on relationship between soil moisture and the radar backscattering cross section from one study site to another and from one parcel to another. 
3) Reflection of a multilayered medium: In this section, we propose the redefinition of the dielectric constant to include the air fractions presented in the soil volume structure, while taking into account the multiscale multilayer description of the soil.

The soil surface in considered as a three-layered medium, where $D$ is the depth of radar signal penetration, as illustrated in Fig. 1. The multilayer soil model is composed of three uniform layers [8]: medium 0 is a half-space, medium 1 with a thickness $d 1$ and a permittivity $\varepsilon 1$, medium 2 , which represents the soil air particles, has $d 2$ as a thickness and $\varepsilon 2$ as a permittivity, medium 3, which represents the soil layer below the depth of radar signal penetration $D(d 1+d 2)$, with a permittivity $\varepsilon 3$ : it's a semi-infinite layer that does not have a thickness.

$E i$ and $E r$ are respectively the incident and reflected radar signal.

We have introduced the multilayer appearance of the soil surface moisture and thereafter, the dielectric constant will be redefined according to the new description to take into consideration the air / soil composition. $\varepsilon_{a p p}$ is an effective permittivity that encompasses the different dielectric permittivities of the three layers.

$\varepsilon_{\text {app }}=\left[v_{\text {sol }} * \varepsilon_{\text {sol }}^{\alpha}+\left(1-v_{\text {sol }}\right) * \varepsilon_{\text {air }}^{\alpha}\right]^{\frac{1}{\alpha}}$

$\alpha=0.5, \varepsilon_{s o l}$ is the dielectric constant of the soil, $\varepsilon_{\text {air }}$ is the dielectric constant of air, and $v_{s o l}$ is the fraction of the soil defined as:

$v_{\text {sol }}=-0.22 \log \left(Z_{s}\right)+0.0058$

$Z_{s}=s^{2} / l$

the volumetric water content is given by $m_{v}$ :

$m_{v}=$

$-5.310^{-2}+2.9210^{-2} \varepsilon_{a p p}-5.510^{-4} \varepsilon_{a p p}^{2}+$

$4.310^{-6} \varepsilon_{a p p}{ }^{3}$

\section{The Impact of Air Pockets Interfaces on RADAR}

Backscattering using Three Layered 2D MLS SPM

To evaluate the effect of the air pockets in the soil, a sensitivity analysis of the backscattering signal is performed.

The backscattering coefficients expressions of three layered multiscale surfaces, for respectively the vertical and parallel polarization $\mathrm{VV}$ and $\mathrm{HH}$ are computed as follow:

$$
\begin{aligned}
& \sigma_{v v}= \\
& 8 k^{4} \sigma_{1}^{2}\left|+\begin{array}{c}
R_{\|} \cos ^{2} \theta \\
\frac{\sin ^{2} \theta\left(1+R_{\|}\right)^{2}}{2}\left(1-\frac{1}{\varepsilon_{r}}\right)
\end{array}\right|^{2} W(2 k \sin \theta, 0) \\
& \sigma_{h h}=8 k^{4} \sigma_{1}^{2}\left|R_{\perp} \cos ^{2} \theta\right|^{2} W(2 k \sin \theta, 0) \\
& W^{n}\left(-2 k_{x}, 0\right)=\frac{2}{\pi} \int_{0}^{\infty} \int_{0}^{\infty}\left(\frac{r_{c}^{i}(\xi, \eta)}{r_{c}^{i}(0,0)}\right)^{n} \cos \left(2 k_{x} \xi\right) d \xi d \eta
\end{aligned}
$$

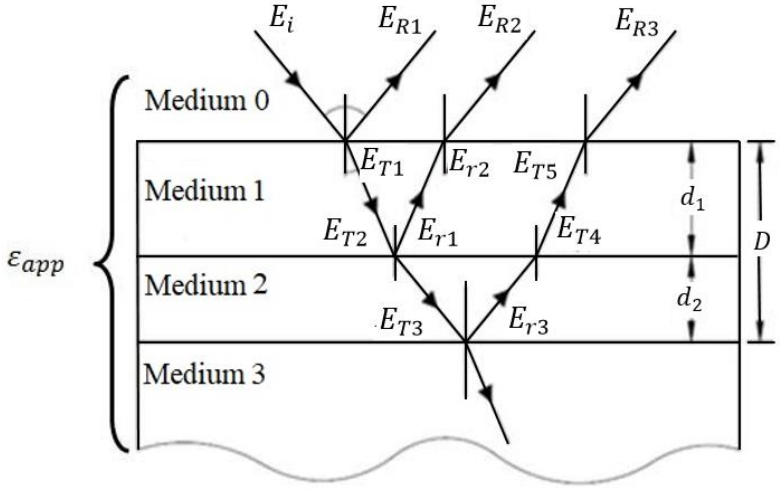

Fig. 1. Multilayered Soil Reflection Model [8].

$\theta$ is the incidence angle. $W^{n}$ is the nth Fourier transform of the autocorrelation function given by Mattia, with $\mathrm{n}=1$ for the SPM model [15] [14] [3] [2].

To better show the adequacy of the three-layered 2D MLS SPM for the description of the soil, we present the table below (Table I) which shows a comparison between our proposed model and recent studies focusing on one and two layers:

The figures show the impact of the air pockets on the backscattering coefficient for the two polarizations VV and $\mathrm{HH}$ in an angular tendency from 20 to 70 degrees.

Fig. 2 and Fig. 3 show the impact of the $v$ parameter related to the fractal dimension on the radar signal backscattering, Fig. 4 and Fig. 5 present the impact of the $\gamma$ parameter related to the standard deviation height on the radar signal backscattering, on both bare soil (solid line) and soil with air pockets (dotted line).

From these two figures (Fig. 2 and Fig. 3) presenting the impact of air pockets on the horizontal dimension of soil roughness $v$, we can note that with an increase of the fractal dimension, the surface becomes smoother, and the value of the backscattering coefficient decreases due to the specular reflection.

Fig. 4 and Fig. 5 show the dependence of the vertical backscattering coefficient on soil roughness vertical dimension $\gamma$. The value of $\sigma_{H H}$ increases with $\gamma$, since an increase in the standard deviation of heights can cause an amplification of the backscattered signal.

TABLE. I. COMPARISON BETWEEN MLS SPM WITH ONE LAYER, TWO LAYERS AND THREE LAYERS FOR ROUGHNESS AND MOISTURE PARAMETERS

\begin{tabular}{|l|l|c|c|}
\hline & $\begin{array}{l}\text { Single layered } \\
\text { MLS SPM [19] }\end{array}$ & $\begin{array}{l}\text { Two-layered } \\
\text { MLS SPM [3] }\end{array}$ & $\begin{array}{l}\text { Three-layered } \\
\text { MLS SPM }\end{array}$ \\
\hline $\begin{array}{l}\text { Relative dielectric } \\
\text { constant } \varepsilon_{r}\end{array}$ & $\varepsilon_{r}$ & $\varepsilon_{1}, \varepsilon_{2}$ & $\varepsilon_{1}, \varepsilon_{2}, \varepsilon_{3} \geq \varepsilon_{r}$ \\
\hline Vertical roughness & $\begin{array}{l}\gamma_{0} \text { related to the } \\
\text { standard } \\
\text { deviation of the } \\
\text { heights }\end{array}$ & $\gamma_{1}, \gamma_{2}$ & $\gamma_{1}, \gamma_{2}$ \\
\hline $\begin{array}{l}\text { Horizontal } \\
\text { roughness }\end{array}$ & $\begin{array}{l}v \text { related to the } \\
\text { fractal } \\
\text { dimension } D\end{array}$ & $v_{1}, v_{2}$ & $v_{1}, v_{2}$ \\
\hline
\end{tabular}




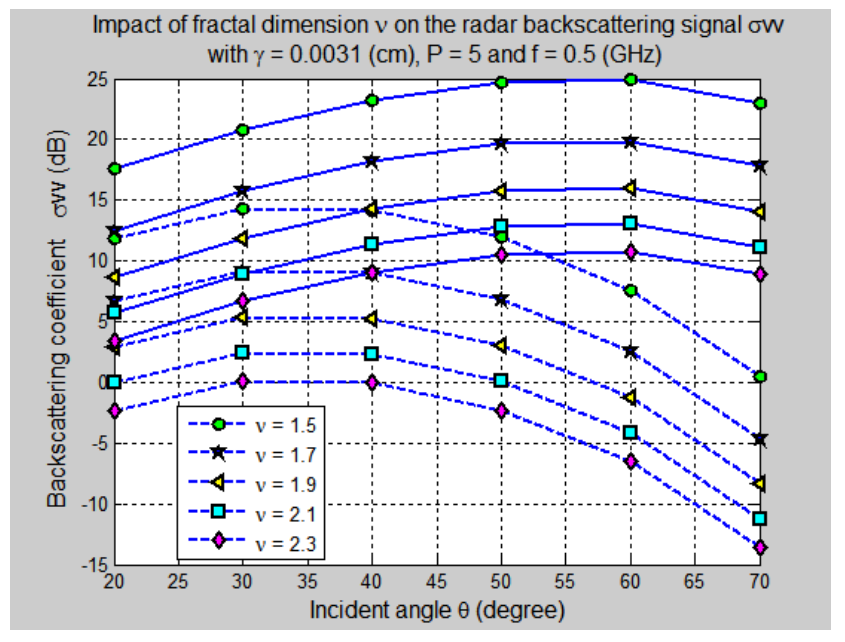

Fig. 2. $\sigma_{V V}$ as a Function of Incident Angle $\theta\left({ }^{\circ}\right)$ for different Values of $v$ with $\gamma=0.0031 \mathrm{~cm}, \mathrm{P}=5$ and $\mathrm{f}=5 \mathrm{Ghz}$.

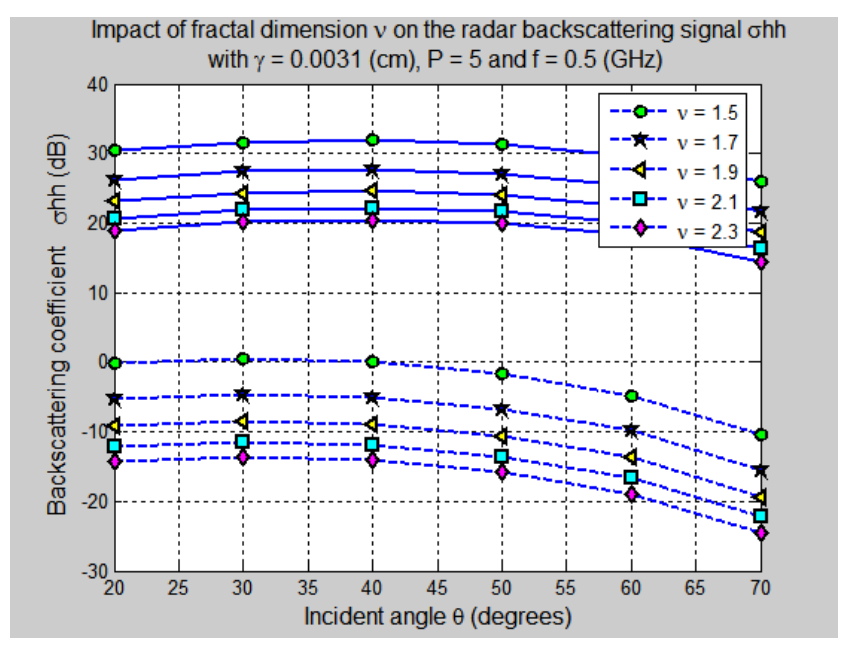

Fig. 3. $\sigma_{H H}$ as a Function of Incident Angle $\theta\left({ }^{\circ}\right)$ for different Values of $v$ with $\gamma=0.0031 \mathrm{~cm}, \mathrm{P}=5$ and $\mathrm{f}=5 \mathrm{Ghz}$.

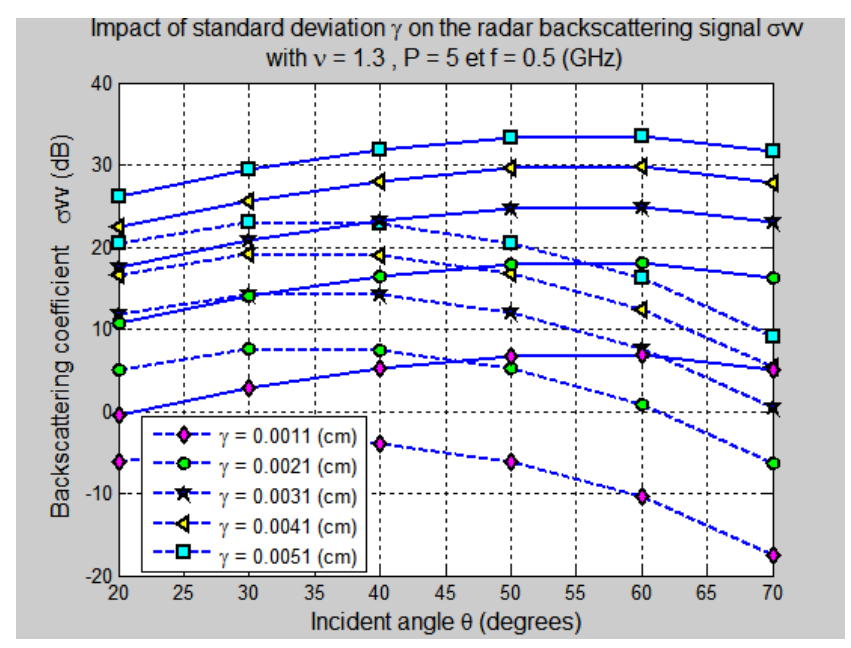

Fig. 4. $\sigma_{V V}$ as a Function of Incident Angle $\theta\left({ }^{\circ}\right)$ for different Values of $\gamma$ with $v=1.3, \mathrm{P}=5$ and $\mathrm{f}=5 \mathrm{Ghz}$.

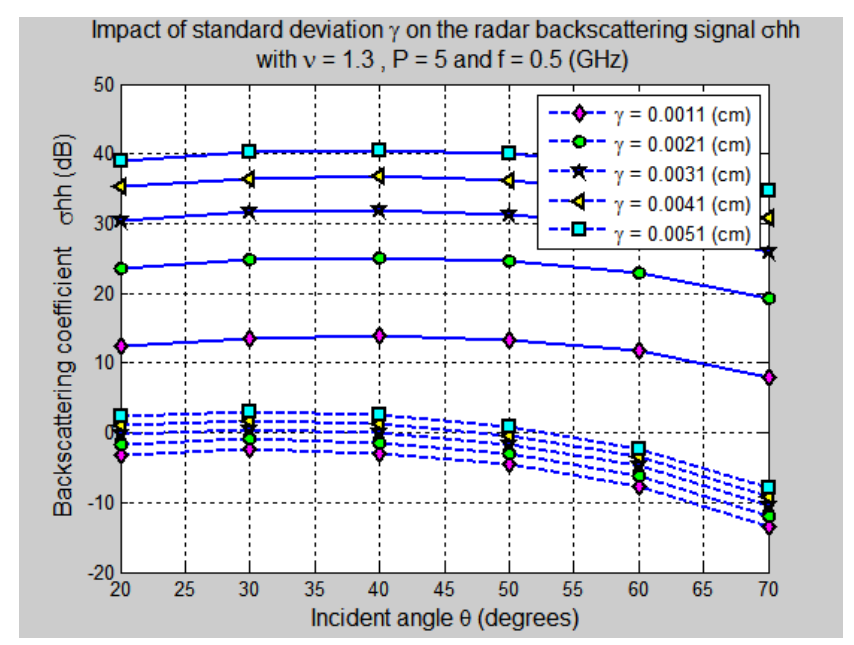

Fig. 5. $\sigma_{H H}$ as a Function of Incident Angle $\theta\left({ }^{\circ}\right)$ for different Values of $\gamma$ with $v=1.3, \mathrm{P}=5$ and $\mathrm{f}=5 \mathrm{Ghz}$.

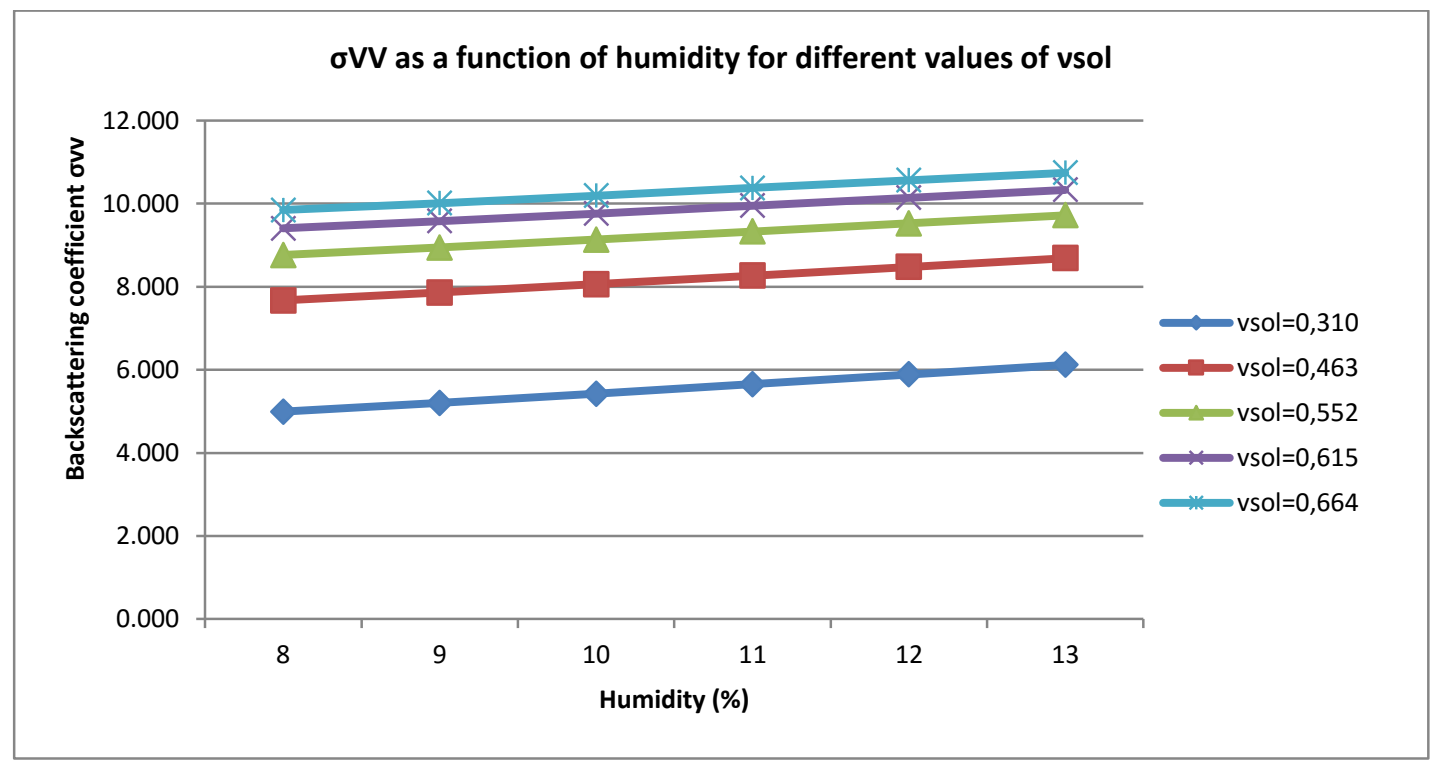

Fig. 6. $\sigma_{V V}$ as a Function of Incident Angle $\theta\left(^{\circ}\right)$ for different values of Humidity. 


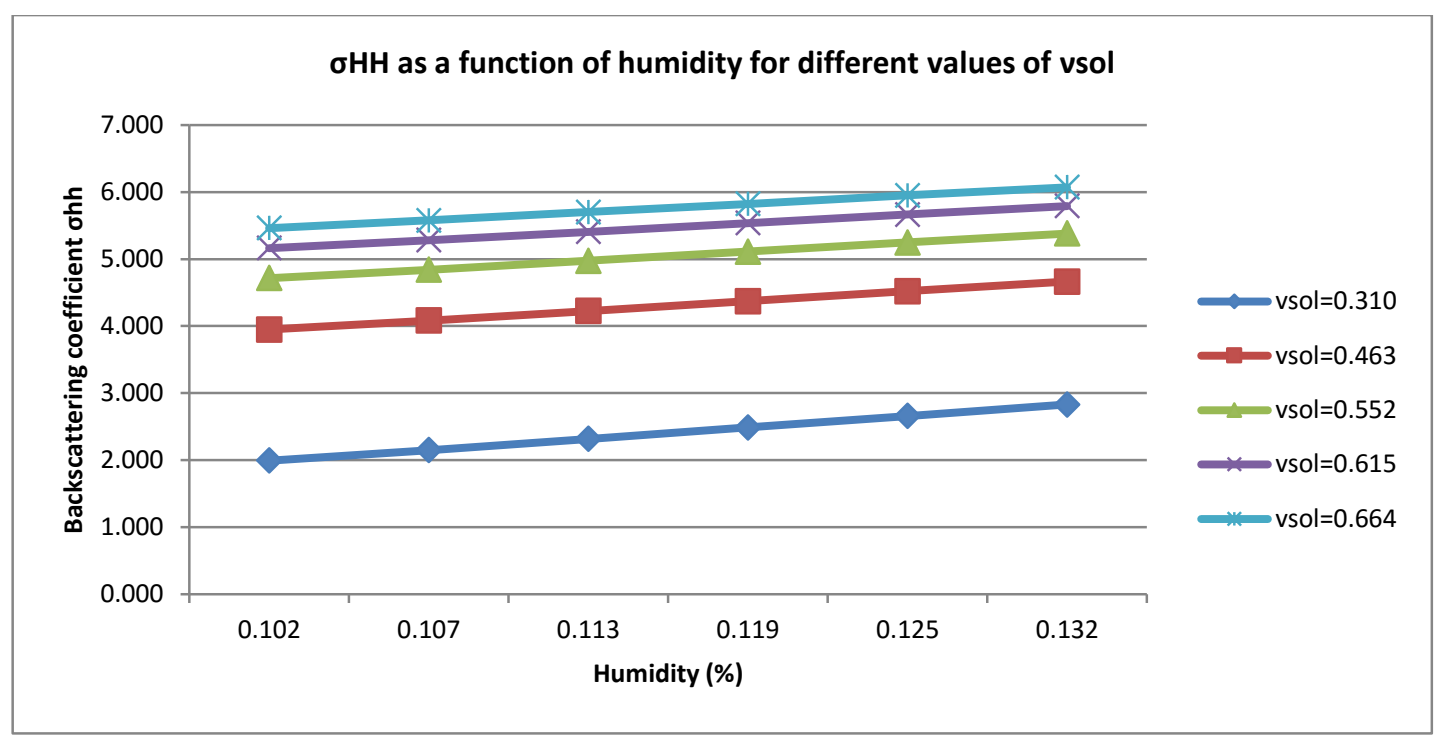

Fig. 7. $\sigma_{H H}$ as a Function of Incident Angle $\theta\left(^{\circ}\right)$ for different Values of Humidity.

In the following two figures (Fig. 6 and Fig. 7), we present the impact of air pockets in soil volume structure on the radar cross section.

Both figures (Fig. 6 and Fig. 7) show that with an increase in the soil moisture value, the amount of the backscattered signal will be higher and the penetration depth of the radar signal will be smaller.

We can note for all simulations, the amount of energy redirected to the radar system increases with the presence of air pockets in the soil texture. This increase reflects the inability areas containing air pockets to capture the emitted radiation and reflect it, and therefore a considerable portion of this radiation is returned to the radar.

\section{Simulation of the Soil Physical Parameters with Volumic Air Pockets}

Our objective is to find an inversion method to best restore the geophysical soil parameters. The number of retrieved soil parameters in the inversion procedure is limited to the number of the same parameters appearing in the direct radar signal simulation solution.

It is necessary to know how to deal with the direct problem, to have an algorithm calculating the bachscattered signal for a given atmosphere conditions and in a given surface geometry.

It is therefore clear that the calculation will be more reliable as the modeling of the backscattering radar signal will be more accurate. It is then necessary to use a precise and efficient inversion method that takes into account the information contained in the studied radar signal.

1) Neural network training: The main objective of the inversion algorithm consists in estimating the geophysical soil parameters namely the multiscale roughness $(v 1, v 2, \gamma 1, \gamma 2)$, and the multiscale moisture $\left(\varepsilon_{a p p}\right)$ from the remote sensing data obtained from the direct model. This algorithm is based on neural networks method witch is trained by learning rules using the backpropagation method. Simulated data sets, based on the adapted SPM surface scattering model, are used to train the neural network $(\mathrm{NN})$.

The inputs correspond to the observation data, while the outputs are the retrieved soil parameters. The adjustment of the internal parameters is done using a database that contains examples of inputs and outputs.

As shown in Fig. 8, backscattering coefficients were introduced as input parameters of the algorithm for different incidence angles measurements ranging from $20^{\circ}$ to $70^{\circ}$ for both horizontal and vertical polarizations ( $\mathrm{HH}$ and $\mathrm{VV}$ ).

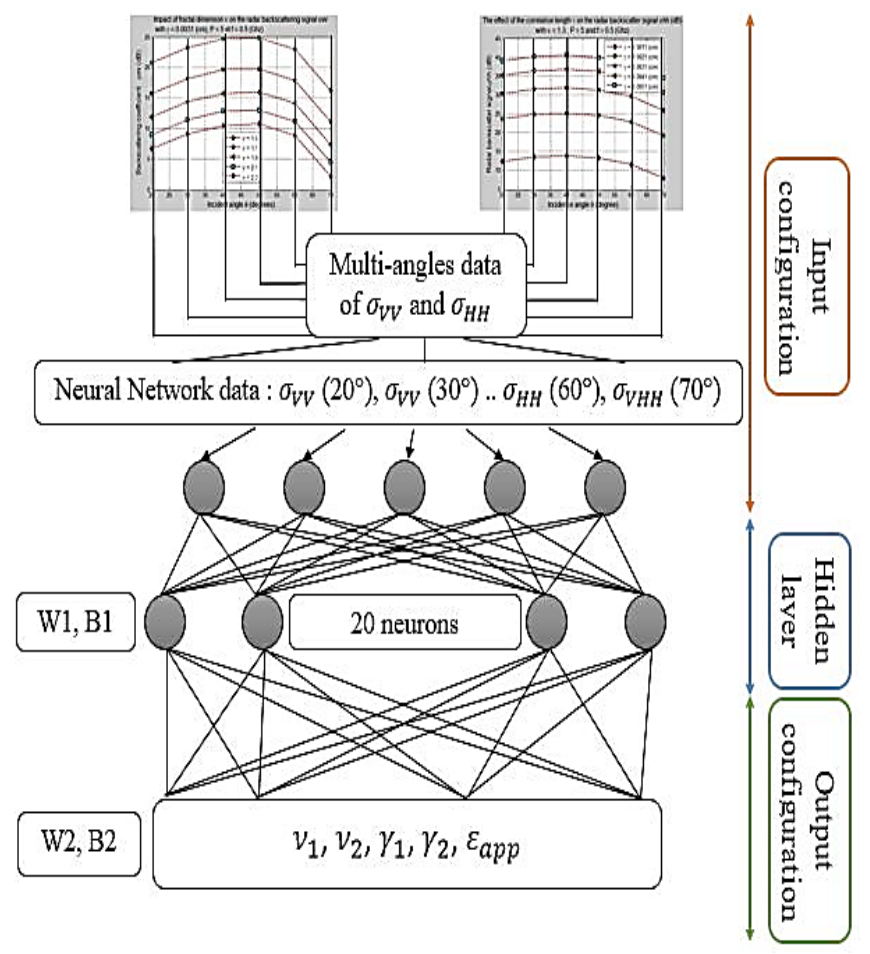

Fig. 8. Neural Network Inversion Method. 
2) NN modelization by Gas: Generally evolutionary algorithms (EA), and genetic algorithms (GA) in particular, are directly derived from faculties of the nature to adapt to the environment by evolving through selection and reproduction. Neural networks (NN) are also a simplified way to simulate the abilities of organisms to adapt to their environment by learning. Simply because nature works well on this way, and successfully, it has been a source of inspiration for many works on hybridization of neural networks (NN) with evolutionary algorithms such as genetic algorithms, hoping that this combination can help resolve problems more effectively that the two methods taken independently.

The first step in allowing NNs to be handled by a GA is to define in which form in the data structure sense the EA will see the $\mathrm{NN}$ as an individual of a population (Fig. 9).

The evolution occurs on chromosomes that represent each of the individuals in a population. The process of natural selection ensures that the most suitable individuals reproduce more often and contribute more to future populations.

During reproduction, the information contained in the individuals of the parents is combined and mixed to produce the individuals of the children. Crossing result may in turn be changed by random perturbations.

For learning, we adopted an input layer with one hundred neurons (relative to populations with $\sigma_{H H}, \sigma_{V V}$ and the incident angle $\theta$ ), a hidden single-layer $\mathrm{NN}$ of twenty neurons, and an output layer of five neurons (relative to the parameters of soil moisture and roughness $\varepsilon_{a p p}, \gamma 1, \gamma 2, v 1, v 2$ ). These latter are derived from a set of weights that are trained with NN.

Both NN and NN-GA hybrid model have been implemented with the classical RPG (Retro-propagation gradient approach) to establish a comparison between them.

We start randomly by generating a population of individuals. To pass from a generation $\mathrm{k}$ to the generation $\mathrm{k}+$ 1 , the following three operations are repeated for all the elements of the population k. Couples of parents P1 and P2 are selected according to their adaptations.

The crossing operator is applied to them with a probability Pc (generally around 0.6) and generates pairs of children C1 and $\mathrm{C} 2$. Other elements $\mathrm{P}$ are selected according to their adaptation.

The mutation operator is applied with probability $\mathrm{Pm}(\mathrm{Pm}$ is generally much lower than $\mathrm{Pc}$ ) and generates mutated individuals P0. The children $(\mathrm{C} 1, \mathrm{C} 2)$ and the P0 mutated individuals are then evaluated before insertion into the new population. Different criteria for stopping the algorithm can be chosen:

- The wished number of generations to execute can be fixed from the beginning. This is what we are tempted to do when we have to find a solution in a limited time.

- The algorithm can be stopped when the population no longer evolves or is no longer fast enough.

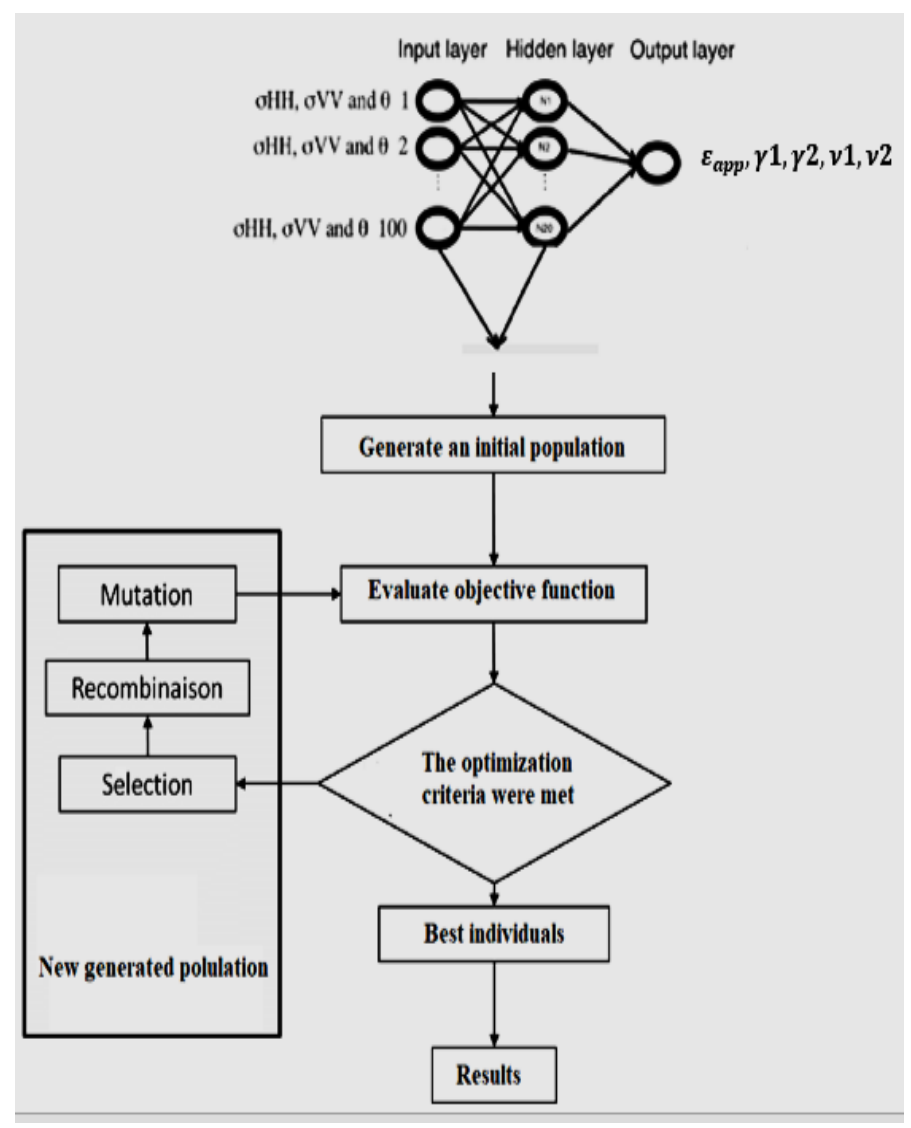

Fig. 9. Proposed Methodology.

The choice of the initial population strongly conditions the speed of the algorithm.

we start by Genome Coding, which is typically based on the storage of multilayer perceptron weights in the form of a matrix W such that $w_{i j}$ corresponds to the weight of the connection from neuron $\mathrm{j}$ to the neuron $\mathrm{i}$. Then simply put end to end each line of the matrix to obtain the genotype of an individual.

The biased roulette principle is used to randomly select pairs of reproducing individuals. This principle is based on the image of a roulette wheel such that the probability of selecting a particular individual is proportional to the value of its adaptation function. Thus, the best individuals will have a higher probability of being selected for reproduction.

3) Inversion algorithm results: The main objective of the inversion algorithm, which is based on a modeled $\mathrm{NN}$ by a GA method, is to retrieve physical soil parameters, namely the multiscale roughness and moisture, from the radar signal.

The obtained results with our approach NN-GA show that the retrieved parameters are improved compared to the inversion model based on NN. Fig. 10, Fig. 11 and Fig. 12 show that the retrieved dielectric constant with NN-GA are better than those obtained by NN. 


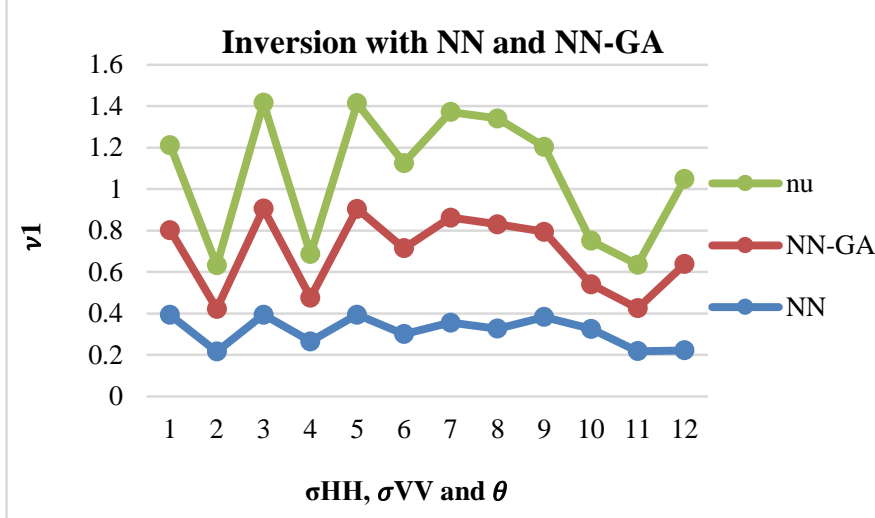

Fig. 10. Optimal Retrieved Fractal Parameter $v 1$.

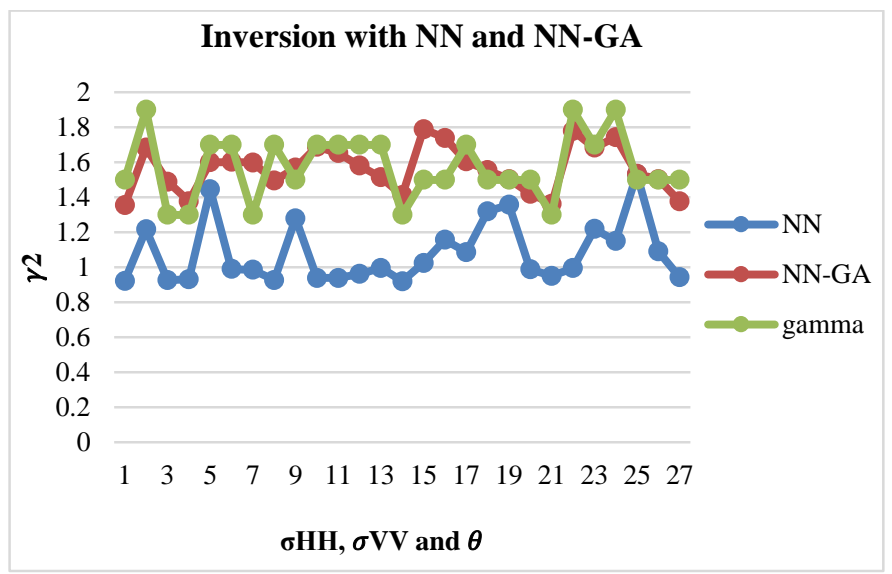

Fig. 11. Optimal Retrieved Standard Deviation $\gamma 2$.

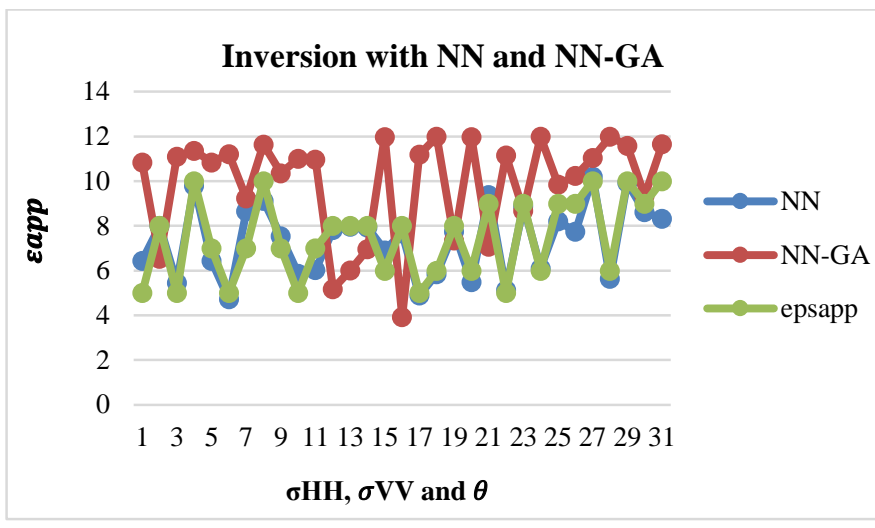

Fig. 12. Optimal Retrieved Dielectric Constant $\varepsilon_{a p p}$.

\section{CONCLUSION}

In this paper we have presented a synergistic method between the Neural Network and the modeled Neural Network by the genetic algorithms, to retrieve the physical parameters in a soil with volumetric air pockets.

The obtained results show that our proposed approach can, with a certain percentage of quadratic error, improve the retrieved parameters compared to the inversion model based on $\mathrm{NN}$.
The main perspectives that follow the work carried out in this work are:

- Use other electromagnetic models in addition to the SPM model, the field of validity of which is restricted, for learning neural networks in order to be able to apply the neuronal inversion method to surfaces of any roughness.

- It will be interesting to apply the multiscale description on other types of surfaces such as maritime surfaces.

\section{REFERENCES}

[1] N. Baghdadi, C. King, A. Bourguignon, A. Remond Potential of ERS and RADARSAT data for surface roughness monitoring over bare agricultural fields: application to catchments in Northern France Int. J. Remote Sens., 23 (2002), pp. 3427-3442.

[2] Farah, L. B, I. R. Farah, R. Bennaceur, Z. Belhadj, and M. R. Boussema, ISoil multiscale roughness parameters and soil moisture retrieval from radar backscattering using a neural network technique," PIERS Proceedings, 367-371, Tokyo, Japan, August 2-5, 2006.

[3] Farah, L. B., I. R. Farah, R. Bennaceur, I. Hosni, and M. R. Boussema, A two layers multiscale bi-dimensional SPM model for the study of radar backscatter behavior on semi-arid soil subsurfaces," PIERS Online, Vol. 6, No. 6, 509-513, 2010.

[4] Mallat, S.G. 1989. Theory of multi-resolution signal decomposition: The Wavelet representation. IEEE Transactions on Pattern analysis and machine intelligence. 1989. Vol. 11, 7, pp. 674-693.

[5] Vidal Vázquez, E., Vivas Miranda, J.G. \& Paz González, A., (2005). Characterizing anisotropy and heterogeneity of soil surface microtopography using fractal models. Ecological Modelling, 182(3-4), pp.337-353.

[6] Ulaby, F. T., M. K. Moore, and A. K. Fung, Microwave Remote Sensing Active and Passive, 3, Artech House, Norwood, M.A., 1986.

[7] Zribi, M, Le Morvan, A et Baghdadi, N . 2008b. Dielectric Constant Modelling with Soil-Air Composition and Its Effect on SAR Radar Signal Backscattered over Soil Surface. Sensors. 2008b. 8, pp. 68106824.

[8] Song, K, Zhou, X et Fan, Y. 2009. Multilayer soil model for improvement of soil moisture estimation using the small perturbation method. Journal of Applied Remote Sensing. 2009. 3. 033567.

[9] Gorrab, A., Zribi, M., Baghdadi, N., Mougenot, B., and Chabaane, Z.L. (2015a). Potential of X-band TerraSAR-X and COSMO-SkyMed SAR data for the assessment of physical soil parameters. Remote Sens. 7, 747-766.

[10] Mohanty, B.P., Cosh, M.H., Lakshmi, V., and Montzka, C. (2017). Soil moisture remote sensing: State-of-the-science. Vadose Zone J. 16.

[11] Wang, H., Méric, S., Allain, S., and Pottier, E. (2014). Adaptation of Oh Model for soil parameters retrieval using multi-angular RADARSAT-2 datasets. J. Surv. Mapp. Eng. 2, 65-74.

[12] Chow V.T., Maidment D.R., Mays L.W. Applied hydrology. McGrawHill Inc.; New York: 1988.

[13] Wagner W., Blöschl G., Pampaloni P., Calvet J.-C., Bizzarri B., Wigneron J.-P., Kerr Y. Operational readiness of microwave remote sensing of soil moisture for hydrologic applications. Nordic Hydrology. 2007;38:1-20.

[14] Davidson M.W.J., Le Toan T., Mattia F., Satalino G., Manninen T., Borgeaud M. On the characterization of agricultural soil roughness for radar remote sensing studies. IEEE Trans. Geosci. Remote Sens. 2000;38:630-640.

[15] Mattia F., Le Toan T. Backscattering properties of multiscale rough surfaces. Journal of Electromagnetic Waves and Applications. 1999;13:491-526.

[16] Borrelli, P., D. A. Robinson Larissa, R. Fleischer, E. Lugato, C. Ballabio, C. Alewell, K. Meus-burger, S. Modugno, B. SchÄutt, V. Ferro, V. Bagarello, K. Van Oost, L. Montanarella, and P. Panagos, "An assessment of the global impact of 21st century land use change on soil 
erosion", Nature Communications, Vol. 8, Article number: 2013, December 08, 2017.

[17] Oh, Y. 2004. Quantitative retrieval of soil moisture content and surface roughness from multipolarized radar observations of bare soil surfaces. IEEE Transactions on Geoscience and Remote Sensing. 2004. Vol. 42, 3 , pp. $596-601$.
[18] Srivastava, H.S, et al. 2003. Use of multiincidence angle of radarsat-1 SAR data to incorporate the effect of surface roughness in soil moisture estimation. IEEE Transactions on Geosciences and Remote Sensing. 2003. Vol. 41, 7, pp. 1638-1640.

[19] Bennaceur, L, Boussema, M.R et Belhadj, Z. 2005. Restitution des paramètres physiques de rugosité et d'humidité. 25 Juin 2005. 